\title{
Effects of matrine on oval cell-mediated liver regeneration and expression of RBP-JK and HES1
}

\author{
ZHI-YUN YANG, LI WANG, YI-XIN HOU and XIAN-BO WANG \\ Department of Traditional Chinese Medicine, Beijing Ditan Hospital, Capital Medical University, Beijing 100015, P.R. China
}

Received September 22, 2012; Accepted January 3, 2013

DOI: $10.3892 / \mathrm{mmr} .2013 .1398$

\begin{abstract}
In the present study a rat model of oval cell-mediated liver regeneration was constructed to examine the molecular mechanisms of matrine in oval cell-mediated liver regeneration and the effects of matrine on hepatic function and the expression of OV6 protein and recombination signal sequence-binding protein $\mathrm{J}_{\kappa}\left(\mathrm{RBP}-\mathrm{J}_{\kappa}\right)$ and HES1 mRNA. A total of 48 Sprague Dawley rats were equally and randomly assigned to two groups. The model group underwent oval cell-mediated liver regeneration, whereas the matrine group underwent oval cell-mediated liver regeneration and received oral gavage of matrine. Expression of OV6 protein was tested by immunohistochemistry and RBP-J $\kappa$ and HES1 mRNA expression was determined by reverse-transcription polymerase chain reaction. Recovery of hepatic function was faster in the matrine group compared with the model $(\mathrm{P}<0.05)$. OV6 protein, RBP-J $\kappa$ and HES1 mRNA expression levels were lower in the matrine than the model group $(\mathrm{P}<0.05)$. Matrine promotes oval cell-mediated liver regeneration through downregulation of the RBP-JK-HES1 signaling pathway.
\end{abstract}

\section{Introduction}

Oval cell-mediated liver regeneration has been a focus of hepatic stem cell studies in recent years. The liver has a notable regeneration capacity and under normal conditions damaged liver tissue is repaired through the proliferation of mature hepatocytes. When the ability of hepatocytes to divide and replace the damaged tissue is compromised, oval cells become activated. These cells then proliferate and differentiate into mature liver cells, thereby aiding liver regeneration. As a result, hepatic stem cell therapy is currently considered as a new approach to liver disease treatment (1-3). However, the safe use of hepatic stem cells must also be considered due

Correspondence to: Professor Zhi-Yun Yang or Professor Xian-Bo Wang, Department of Traditional Chinese Medicine, Beijing Ditan Hospital, Capital Medical University, 8 Jingshun Dong Road, Chaoyang, Beijing 100015, P.R. China

E-mail: zhiyunyang@yeah.net

E-mail: wangxianbo638@163.com

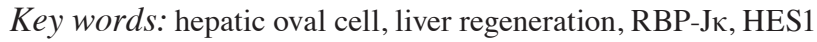

to the possibility of malignant transformation $(4,5)$. Therefore, regulation of the activation and expansion of liver progenitor cells has also been analyzed. In addition, studies on pharmacologically effective agents from natural products associated with low toxicity have also been performed.

Matrine is one of the main alkaloid components extracted from the Sophora root and has a molecular formula of $\mathrm{C}_{15} \mathrm{H}_{24} \mathrm{~N}_{2} \mathrm{O}$ (6). Studies have revealed that matrine protects the stability of liver cell membranes, inhibiting proliferation of mesenchymal cells and regulating satellite cells. In addition, the compound exhibits anti-inflammatory $(7)$, antiviral $(8,9)$, immunoinhibitory, antifibrotic (10) and antidiarrheal (11) effects. However, its role in oval-cell mediated regeneration remains unclear. Therefore, the aims of the present study were to determine the effects of matrine on liver regeneration and elucidate its molecular mechanisms.

The signals mediating cellular specification are produced by adjacent or distant cells and these signals are often received by the cell simultaneously. Experimental evidence has demonstrated that Notch receptors and ligands are required for mammalian development and growth in a number of organ systems, including the liver (12-14). In particular, the Notch signaling pathway is key to regulating the differentiation of various stem cells. However, only a limited number of studies have been conducted on hepatic stem cells. Therefore, in this study, the potential role of Notch signaling in the regulation of oval cell-mediated liver regeneration was examined. An oval-cell mediated liver regeneration model was constructed and treated with matrine. In addition, the expression of recombination signal sequence-binding protein $\mathrm{J}_{\kappa}\left(\mathrm{RBP}-\mathrm{J}_{\kappa}\right)$ and HES1, key molecular components of the Notch signaling pathway in regenerated liver tissue, was determined. The results of the current study are likely to demonstrate the effects of matrine in the proper differentiation and growth of oval cells regulated by Notch signaling.

\section{Materials and methods}

Animal models. Male Sprague Dawley (SD) rats (weight, $200 \pm 20 \mathrm{~g}$ ) were used. All procedues were performed in strict accordance with the Guide for the Care and Use of Laboratory Animals of the National Institutes of Health. The animal use protocol was reviewed and approved by the Institutional Animal Care and Use Committee of Beijing Ditan Hospital (Beijing, China). 
Table I. Primer sequences used for RT-PCR.

\begin{tabular}{|c|c|c|c|c|}
\hline Gene & Primer sequence & $\begin{array}{c}\text { Annealing } \\
\text { temperature }\left({ }^{\circ} \mathrm{C}\right)\end{array}$ & Cycles & $\begin{array}{l}\text { PCR product } \\
\text { length (bp) }\end{array}$ \\
\hline RBP-J & $\begin{array}{l}\text { 5'-CCA ATT TCA GGC CAC TCC A-3' } \\
\text { 5'-CTC TAC ATC CCC AAA CCA CAC TC-3' }\end{array}$ & 54.2 & 35 & 253 \\
\hline HES1 & $\begin{array}{l}\text { 5'-CAA CAC GAC ACC GGA CAA ACC-3' } \\
\text { 5'-AGT GCG CAC CTC GGT GTT AAC-3' }\end{array}$ & 51.8 & 35 & 349 \\
\hline$\beta$-actin & $\begin{array}{l}\text { 5'-GCC ATG TAC GTA GCC ATC CA-3' } \\
\text { 5'-GAA CCG CTC ATT GCC GAT AG-3' }\end{array}$ & & & 375 \\
\hline
\end{tabular}

$\mathrm{RBP}-\mathrm{J}_{\kappa}$, recombination signal sequence-binding protein $\mathrm{J} \kappa$; RT-PCR, reverse-transcription polymerase chain reaction.

Male SD rats were randomly divided into two groups, model and matrine (both $\mathrm{n}=24$ ). Rats were fed with pellet chow and provided access to water ad libitum. Rats were maintained in a temperature-controlled room with a $12-\mathrm{h}$ light/dark illumination cycle. All rats received daily oral gavage of N-2-acetylaminofluorene (2-AAF; Sigma-Aldrich, St. Louis, MO, USA) at a dosage of $15 \mathrm{mg} / \mathrm{kg}$ for 1 week prior to and 2 weeks following partial hepatectomy $(\mathrm{PH})$ to inhibit hepatocyte proliferation. The 2-AAF was dissolved in dimethyl sulfoxide (Sigma-Aldrich). The matrine group also received daily oral gavage of matrine (batch no. 110780-201012, China Drugs and Biological Products Inspection Institute, Beijing, China) at a dosage of $20 \mathrm{mg} / \mathrm{kg}$. Matrine was reconstituted in distilled water. Following 1 week of daily gavage, all rats were anesthetized and two-thirds PH was performed by surgically removing the left and median liver lobes. No dosing was performed on the day of the surgery. Three rats from each group were sacrificed on days 1,3,7 and 14 following PH. Formalin-fixed and paraffin-embedded serial liver tissue sections $(4 \mu \mathrm{m})$ were used for immunohistochemistry and reverse-transcription polymerase chain reaction (RT-PCR).

Survival rate test. The number of rats that survived in each group was counted on days 1, 4, 7, 10, 14 and 21 of analysis and the survival rate was calculated for each group.

Liver function test. Aminotransferase (ALT) and total bilirubin (TBil) levels, which reflect liver function, were tested on days 1, 4, 7, 10, 14 and 21 of analysis. Blood from the rats tails was used to test for ALT and TBil.

Immunohistochemistry. Paraffin sections of the formalin-fixed liver tissues were stained using a mouse monoclonal antibody against OV6 (MAB 2020; R\&D Systems, Minneapolis, MN, USA), a marker of hepatic oval cells in ductular reactions. Tissue sections were rehydrated using descending concentrations of ethanol. Endogenous peroxidase activity was blocked with $3 \%$ hydrogen peroxide in methanol. Tissues used for OV6 immunohistochemistry were microwaved to boiling for $15 \mathrm{~min}$ in $10 \mathrm{mmol} / 1$ Tris buffer and $1 \mathrm{mmol} / 1$ ethylenediamine tetraacetic acid (EDTA) at pH 9.0 for antigen retrieval. Following antigen retrieval, tissue sections were blocked with $10 \%$ normal serum from the donor species of the secondary antibodies for $15 \mathrm{~min}$ at room temperature and incubated with primary antibodies overnight at $4^{\circ} \mathrm{C}$. The ratio of anti-OV6 dilution was 1:10. Following rinsing with phosphate-buffered solution (PBS), the primary antibody was detected by incubation for $30 \mathrm{~min}$ with biotinylated rabbit anti-mouse immunoglobulin, rinsed further with PBS and incubated with horseradish peroxidase-conjugated streptavidin/biotin complex (85-9843, Histostain Plus kits; Zymed Laboratories Inc., Carlsbad, CA, USA). Peroxidase activity was developed with $0.05 \%$ diaminobenzidine and $0.03 \% \mathrm{H}_{2} \mathrm{O}_{2}$. Finally, sections were counterstained for $5 \mathrm{~min}$ in hematoxylin, dehydrated using graded alcohol and then mounted under glass coverslips.

RNA isolation and RT-PCR. Total RNA from fresh liver tissues of model and matrine groups was extracted with TRIzol according to the manufacturer's instructions at the beginning of the experiment and on days 7, 14 and 21 . RNA ( $1 \mu \mathrm{g})$ was reverse transcribed to cDNA. The number of cycles corresponded to the mid-logarithmic phase for semi-quantitative PCR. Primers were then designed using GenBank sequences (Table I). PCR amplification was performed using PCR Master Mix (Taqman, Takara, Dalian, China) according to the manufacturer's instructions. PCR products were analyzed via electrophoresis (Gel-Pro Analyzer Version 3.0; Media Cybernetics, Inc., Bethesda, MD, USA) on a $1.5 \%$ agarose gel. Results of the semi-quantitative PCR were expressed using the optical density ratio of the value of RBP-JK and HES1 to $\beta$-actin.

Statistical analysis. Data from at least three independent experiments were used for statistical analysis. All results are expressed as mean \pm SD. Measurement data were analyzed using one-way analysis of variances and performed using SPSS v17.0 (SPSS Inc., Chicago, IL, USA). P $<0.05$ was considered to indicate a statistically significant difference.

\section{Results}

Survival rate. The majority of rats had poor appetites, drank and exercised little and appeared dejected on days 1-3 following surgery. One week following PH the characteristics/behavior of the rats had almost returned to those observed prior to surgery. Wounds were re-sutured to ensure improved healing in 3 and 2 rats from the model and matrine groups, 


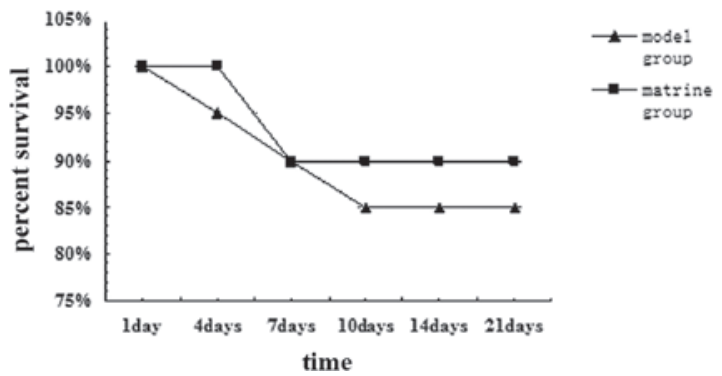

Figure 1. Survival rate of model and matrine groups.

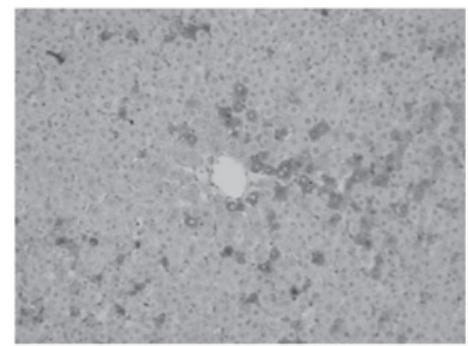

Figure 2. Expression of OV6 in liver tissue of model group at day 3 following partial hepatectomy (magnification, $\mathrm{x} 200$ ).

respectively. The same number of rats from each group died 1 week following surgery. The survival rates of the model group on days 1, 4, 7, 10, 14 and 21 of analysis were 100, 95, $90,85,85$ and $85 \%$, respectively, whereas those of the matrine group were $100,100,90,90,90$ and $90 \%$, respectively. No statistically significant difference was observed between the survival rates of the two groups (Fig. 1).

Liver function recovery. Rats from the model and matrine groups were observed to exhibit the most serious liver impairments at day 1 and 3 following surgery. Liver slowly recovered at day 7 and appeared almost normal by day 14. Compared with the model group, lower ALT and TBil values on days 7, 10, 14 and 21 were identified in matrine rats, indicating that matrine may aid repair of the impaired liver and protect liver function during liver regeneration (Table II).

Proliferation of oval cells. Oval cells were found in the ductular area on day 1 following PH. Compared with mature hepatocytes, oval cells were observed to exhibit reduced volume, higher ratios of nucleus to cytoplasm, round- or oval-shaped nuclei and antibody OV6 expression. The number of oval cells increased with time. OV6-expressing cells were found to be distributed along the ductular to the parenchymal regions of the liver on days 3-7. Cell numbers peaked at day 7 and then decreased. A marked decrease was noted at day 14 following surgery, however, OV6-expressing cells were noted only in the ductular region. Compared with model, fewer OV6-expressing cells were identified in the matrine group (Figs. 2-5 and Table III).

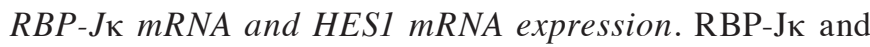
HES1 mRNA expression was analyzed on days 1, 7, 14 and 21 of analysis. Expression peaked at day 7 of the experiment (one day following PH) and decreased over time in the model group. On day 1 of the experiment, no significant difference 
Table III. Number of OV6-expressing cells in the liver tissue of model and matrine groups at various days.

\begin{tabular}{|c|c|c|c|c|c|c|c|c|}
\hline \multirow[b]{2}{*}{ Group } & \multicolumn{2}{|c|}{ Day 1} & \multicolumn{2}{|c|}{ Day 7} & \multicolumn{2}{|c|}{ Day 14} & \multicolumn{2}{|c|}{ Day 21} \\
\hline & $\mathrm{n}$ & Cell number & $\mathrm{n}$ & Cell number & $\mathrm{n}$ & Cell number & $\mathrm{n}$ & Cell number \\
\hline Model & 6 & $8.3 \pm 3.8$ & 6 & $26.3 \pm 9.1$ & 6 & $38.7 \pm 16.8$ & 3 & $20.2 \pm 8.3$ \\
\hline Matrine & 6 & $7.9 \pm 3.9$ & 6 & $18.2 \pm 8.3^{\mathrm{a}}$ & 6 & $21.1 \pm 9.4^{\mathrm{a}}$ & 4 & $11.0 \pm 4.7^{\mathrm{a}}$ \\
\hline
\end{tabular}

${ }^{\mathrm{a}} \mathrm{P}<0.01$, vs. model group.

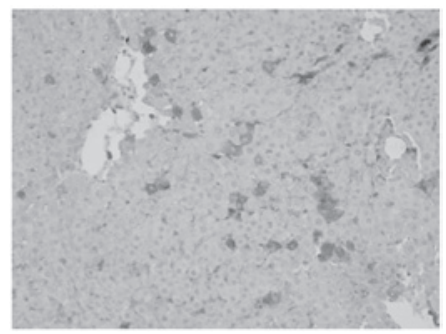

Figure 3. Expression of OV6 in liver tissue of matrine group at day 3 following partial hepatectomy (magnification, $\mathrm{x} 200$ ).

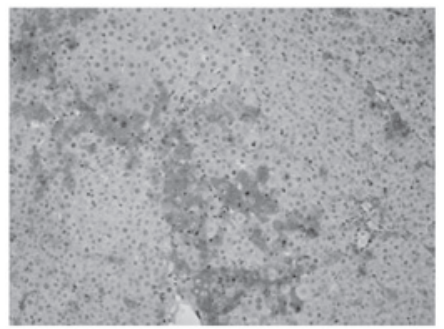

Figure 4. Expression of OV6 in liver tissue of model group at day 14 following partial hepatectomy (magnification, x200).

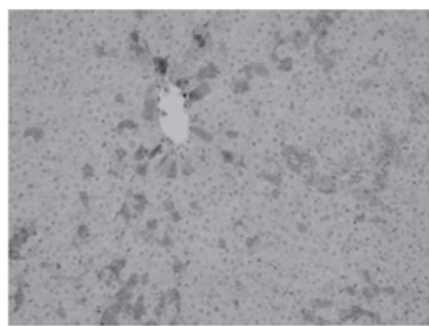

Figure 5. Expression of OV6 in liver tissue of matrine group at day 14 following partial hepatectomy (magnification, x200).

was observed between RBP-J $\kappa$ and HES1 mRNA expression of the model and matrine groups. By contrast, on days 7, 14 and 21 of the experiment, a significant difference was found in RBP-J $\kappa$ and HES1 mRNA expression between the groups $(\mathrm{P}<0.05)$. The matrine trial group experienced a larger decrease in expression levels (Figs. 6 and 7).

\section{Discussion}

Damaged liver tissue is mainly rebuilt through the proliferation of mature hepatocytes and extracellular matrix. However,

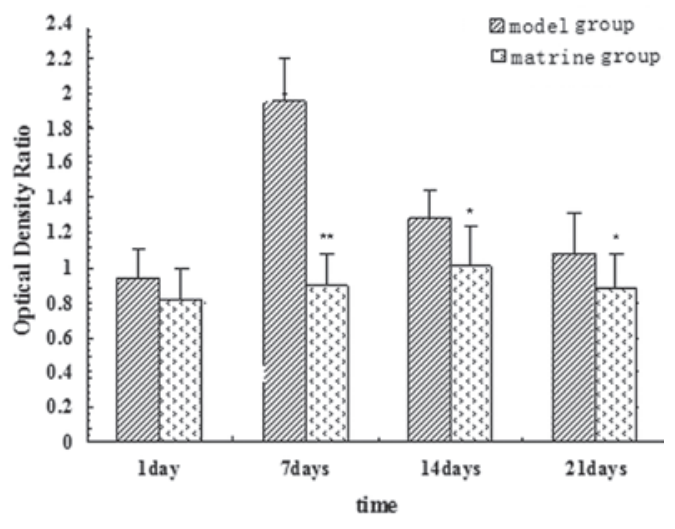

Figure 6. Effects of matrine on expression of RBP-JK mRNA during oval cell-mediated liver regeneration. ${ }^{*} \mathrm{P}<0.05$ and ${ }^{* *} \mathrm{P}<0.01$, vs. model group. RBP-J $\mathrm{K}$, recombination signal sequence-binding protein $\mathrm{J} \kappa$.

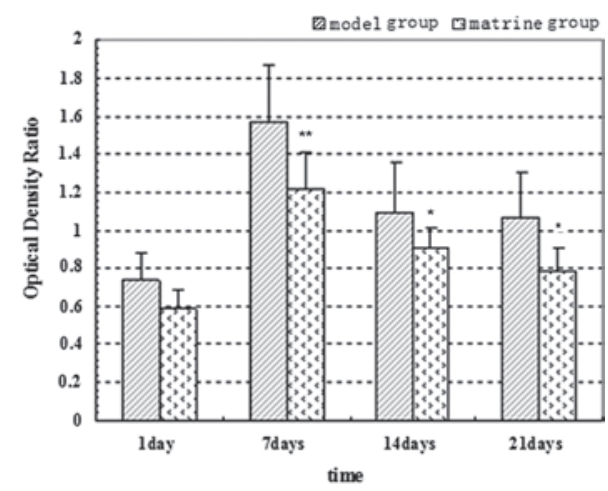

Figure 7. Effects of matrine on expression of HES1 mRNA during oval cell-mediated liver regeneration. ${ }^{*} \mathrm{P}<0.05$ and ${ }^{* *} \mathrm{P}<0.01$, vs. model group.

the ability of the liver to regenerate becomes compromised if it suffers excess damage or if the proliferation process of hepatocytes is blocked. Oval cell activation and proliferation serve as sources of tissue repair and cell replenishment, aiding liver regeneration. The proliferation and differentiation of oval cells to mature hepatocytes and cholangiocytes occurs in a specific micro-environment of extracellular matrix and defined signaling pathways. Therefore, it is not only difficult to determine the mechanisms of proliferation and differentiation of oval cells, but also which drugs induce differentiation. These are the two main issues in the study of liver stem cells (14).

In the present study, proliferation and differentiation of oval cells in oval cell-mediated liver regeneration in $2-\mathrm{AAF} / \mathrm{PH}$ rat models was analyzed. The proliferation of mature hepa- 
tocytes was inhibited by 2-AAF. Activation, proliferation and differentiation of oval cells occurred shortly following removal of two-thirds of the liver. An oval cell-mediated liver regeneration model was successfully constructed and the survival rate of the rats was $>85 \%$ in the model and matrine groups. No statistically significant difference in survival rate was identified between the groups. Bleeding and serious infections were minimized during the surgical procedure, leading to improved recovery rates in the majority of the rats, therefore verifying the high regenerative ability of the liver. Changes in liver function were also analyzed and ALT and TBil were observed to alter over time. The results demonstrate that ALT and TBil levels in the model group were increased at day 1 following surgery, peaked at day 3 and then decreased at day 7. Compared with the model group, the trial group exhibited improved liver function on days 1, 3, 7 and 14, particularly on days 3 and 7.

Results indicate that matrine may aid liver damage recovery, consistent with previous studies. The mechanism by which matrine mediates this function may be associated with its ability to clear free radicals and induce the chondriosome drug metabolism enzyme of hepatocytes $(10,11)$. The current study indictates that matrine regulates oval cell-mediated liver regeneration through its effects on specific signaling pathways that implement this mechanism.

In this study, proliferation of oval cells was revealed to occur in accordance with the recovery of liver function during regeneration of liver. Results indicate that liver function was most impaired at days 3 and 7 following surgery, whereas oval cell proliferation was the highest at day 7 . The proliferation of oval cells decreased following the recovery of liver function. Compared with the model, the matrine trial group exhibited a reduced number of proliferating oval cells on days 3, 7 and 14 , indicating that matrine inhibits the excessive proliferation of oval cells. When proliferation of mature hepatocytes was blocked, oval cells were induced to proliferate and differentiate in response to the inhibition. During the process of oval cell-mediated liver regeneration, matrine inhibited excessive proliferation of oval cells, enabling regeneration of liver tissue and the recovery of liver function to proceed normally. The mechanism of the effects of matrine on oval cell-mediated liver regeneration remains to be studied. In addition, it remains unclear whether matrine directly induces oval cells to differentiate into mature hepatocytes to repair the damaged liver tissue or whether it affects differentiation, migration and proliferation indirectly by regulating the extracellular matrix of the microenvironment $(15,16)$.

The Notch-RBP-Jא signaling pathway is important for maintenance of the characteristics and cell fate specification of various types of stem cells. Alteration of signaling pathway activity or unregulated expression of its ligands and receptors is associated with specific diseases. RBP- $\mathrm{J}_{\kappa}$ is a key protein of the Notch signaling pathway, and as a kind of DNA combination protein, it is combined with specific DNA sequence to induce gene priming $(17,18)$. A number of studies have demonstrated that upregulation of Notch signaling inhibits embryo stem cells from differentiating into a number of specialized cell types, including neural and insulin B cells $(19,20)$. With respect to liver stem cell regulation, previous studies have reported high expression of Notch-1 protein in bone mesen- chymal stem cells and that this expression decreases following bone mesenchymal stem cell differentiation into mature hepatocytes $(21,22)$.

Results of the present study are in agreement with those obtained in previous studies. Serious damage to the liver led to proliferation of a large number of oval cells, accompanied by upregulation of RBP-J $\kappa$ and HES1 mRNA expression in the liver tissue. As the liver was repaired, proliferation of oval cells decreased and expression of RBP-JK and HES1 mRNA was downregulated, indicating that upregulation of RBP-J $\kappa$ and HES1 mRNA is a pivotal process during stem cell maintenance of oval cells. Compared with model, the matrine group was identified to express low levels of RBP-JК and HES1 mRNA on days 1, 7 and 14 following surgery, indicating that downregulation of the Notch-RBP-JK signaling pathway is closely associated with oval cell-mediated liver regeneration.

The current study demonstrates that matrine inhibits excessive proliferation of oval cells, protects liver function and promotes liver regeneration through downregulation of the Notch-RBP-J $\kappa$ signaling pathway. These results are consistent with the hypothesis that Chinese medicine may contribute to advancement of liver regeneration. However, there may be more signaling pathways involved in this procdure. Therefore, additional studies should be performed.

\section{Acknowledgements}

This study was supported by the National Natural Science Foundation of China (no. 30873423), the Youth Science Research Program of Traditional Chinese Medicine Association of Beijing (QN2010-7) and the Traditional Chinese Medicine Personnel Training Plan.

\section{References}

1. Kuhlmann WD and Peschke P: Hepatic progenitor cells, stem cells and AFP expression in models of liver injury. Int J Exp Pathol 87: 343-359, 2006.

2. Libbrecht L and Roskams T: Hepatic progenitor cells in human liver diseases. Semin Cell Dev Biol 13: 389-396, 2002.

3. Santoni-Rugiu E, Jelnes P, Thorgeirsson SS and Bisgaard HC: Progenitor cells in liver regeneration: molecular responses controlling their activation and expansion. APMIS 113: 876-902, 2005.

4. Lee JS, Heo J, Libbrecht L, et al: A novel prognostic subtype of human hepatocellular carcinoma derived from hepatic progenitor cells. Nat Med 12: 410-416, 2006.

5. Shupe T and Petersen BE: Evidence regarding a stem cell origin of hepatocellular carcinoma. Stem Cell Rev 1: 261-264, 2005.

6. Li Y, Wang B, Zhou C and Bi Y: Matrine induces apoptosis in angiotensin II-stimulated hyperplasia of cardiac fibroblasts: effects on Bcl-2/Bax expression and caspase-3 activation. Basic Clin Pharmacol Toxicol 101: 1-8, 2007.

7. Cheng H, Xia B, Zhang L, et al: Matrine improves 2,4,6-trinitrobenzene sulfonic acid-induced colitis in mice. Pharmacol Res 53: 202-208, 2006.

8. Liu J, Zhu M, Shi R and Yang M: Radix Sophorae flavescentis for chronic hepatitis B: a systematic review of randomized trials. Am J Chin Med 31: 337-354, 2003.

9. Long Y, Lin XT, Zeng KL and Zhang L: Efficacy of intramuscular matrine in the treatment of chronic hepatitis B. Hepatobiliary Pancreat Dis Int 3: 69-72, 2004.

10. Zhang JP, Zhang M, Zhou JP, et al: Antifibrotic effects of matrine on in vitro and in vivo models of liver fibrosis in rats. Acta Pharmacol Sin 22: 183-186, 2001.

11. Ai J, Gao HH, He SZ, Wang L, Luo DL and Yang BF: Effects of matrine, artemisinin, tetrandrine on cytosolic $\left[\mathrm{Ca}^{2+}\right]_{\mathrm{i}}$ in guinea pig ventricular myocytes. Acta Pharmacol Sin 22: 512-515, 2001. 
12. Baldi A, de Falco M, de Luca L, et al: Characterization of tissue-specific expression of Notch-1 in human tissues. Biol Cell 96: 303-311, 2004

13. Fausto $\mathrm{N}$ and Campbell JS: The role of hepatocytes and oval cells in liver regeneration and repopulation. Mech Dev 120: 117-130, 2003.

14. Sautin YY, Jorgensen M, Petersen BE, Saulnier-Blache JS, Crawford JM and Svetlov SI: Hepatic oval (stem) cell expression of endothelial differentiation gene receptors for lysophosphatidic acid in mouse chronic liver injury. J Hematother Stem Cell Res 11: 643-649, 2002.

15. Fiegel HC, Havers J, Kneser U, et al: Influence of flow conditions and matrix coatings on growth and differentiation of three-dimensionally cultured rat hepatocytes. Tissue Eng 10: 165-174, 2004.

16. Leite AR, Correa-Giannella ML, Dagli ML, et al: Fibronectin and laminin induce expession of islet cell markers in hepatic oval cells in culture. Cell Tissue Res 327: 529-537, 2007.
17. Hansson EM, Lendahl U and Chapman G: Notch signaling in development and disease. Semin Cancer Biol 14: 320-328, 2004.

18. Stoekhausen MT, Kristofersen K and Poulsen HS: The functional role of Notch signaling in human gliomas. Neuro Oncol 12: 199-211, 2010.

19. Kobayashi T and Kageyama R: Hesl regulates embryonic stem cell diferentiation by suppressing Notch signaling. Genes Cells 15: 689-698, 2010.

20. Murtaugh LC, Stanger BZ, Kwan KM and Melton DA: Notch sin signaling controls multiple steps of pancreatic differentiation. Proc Natl Acad Sci USA 100: 14920-14925, 2003.

21. Okumoto K, Saito T, Hattori E, et al: Differentiation of bone marrow cells into cells that express liver-specific genes in vitro implication of the Notch signals in differentiation. Biochem Biophys Res Commun 304: 691-695, 2003.

22. Schwartz RE, Reyes M, Koodie L, et al: Multipotent adult progen itor cells from bone marrow differentiate into functional hepatocyte-like cells. J Clin Invest 109: 1291-1302, 2002. 